\title{
Identification of the Place and Materials of Knocking Objects in Flow Induced Vibration
}

\author{
Tibor Dobján*†, Szilveszter Pletl ${ }^{\dagger}$, Tamás Deák*, \\ László Doszpod*, and Gábor Pór*
}

\begin{abstract}
Flow induced vibration can be found and identified by acoustic methods. Using acoustic sensors, the first task, the event detection has been solved using the sequential probability ratio test after autoregressive filtering the measured signal. The LABView program and actual test of the event recognition technique are presented. The signals were recorded in a $100 \mathrm{~m}$ long test loop having artificially placed flow induced vibrating objects hitting the wall. Time delay between the fronts of detected events has been used to localize the actual place of the acoustic source. The recognition of the material of the knocking object is based traditionally on the spectrum estimation. However, this is rather time consuming task by naked eyes. We are proposing to introduce the skeleton method for event identification.
\end{abstract}

Keywords: Autoregressive filtering, Sequential Probability Ratio Test, IAEA Benchmark measurement, event detection, skeleton method

\section{Introduction}

Many industrial systems contain pipes with fluid flow either for transmitting materials or for cooling purposes. If solid parts of the system are detached or loosened they may go to chaotic or deterministic motion due to forces gained from the flow energy. It is also quite common when either a disattached part of the equipment or just a forgotten object after maintenance work remains in the pipes causing so called loose parts, which might be even dangerous for the given industrial system. If a loose part knocks on the inner surface of the tube (or other compartment) then audible events are generated. These are surface waves traveling on the metal surfaces. The place of the knocks and the knocking material are crucial from the point of view of the fate of the given industrial objects. Therefore detection of

\footnotetext{
*Hungarian Acoustic Industrial Diagnostic Laboratory, College of Dunaújváros,

E-mail: \{dobjan.tibor, doszpod.laszlo, por.gabor\}@mail.duf.hu, thomasckik@gmail.com

${ }^{\dagger}$ Department of Technical Informatics, University of Szeged,

E-mail: pletl@inf.u-szeged.hu
} 
the event, finding of its place and identification of knocking material have primary importance from the point of view of the safety and maintenance of the system.

Flow via piping systems in industry may excite vibrations. They are called flow-induced vibration. Some of those vibrations are expressed as eigenvalues of the pipes filled with streaming liquid. In some other cases solid parts transported by the liquid phase may also emit sound. These sounds are regarded as background noise in our case.

The task is to notice sound emissions due to loose parts on a relatively large background level and to identify their origin $[1,2,3,4]$. In this paper we investigate the following areas. Improvement of the identification of the event recognition using autoregressive (AR) modeling based filtering and sequential probability ratio test (SPRT). The test measurements are described in Section 1. Data processing has been realized in LABVIEW. This was a very important preparation for identifying the event, since the correct event selection is the basic for that. The method and the program test are presented in Section 2.

To give a hint on the material of the knocking objects first we estimated the auto power spectral density function (APSD). Then we divided the total frequency band into high frequency part and low frequency part. It was shown, that the ratio of these partial root mean square (RMS) values are different for knocking object of different materials. We present the first results of division of APSD into several parts. It can be clearly seen, that this may improve the identification. (See Section 4.) Besides the identification the source localization is also important (Section 3.2.).

In event identification we are going to use skeleton method, neutral network and later a specifically designed expert system, which has its own library based on experiments (Section 4.).

\section{Measurement}

\subsection{Test section}

We carried out measurement sampling signals of accelerometers in frequency range from $1 \mathrm{kHz}$ up to $50 \mathrm{kHz}$. The lower frequencies may have very high industrial noise, while the frequency range above audible $16 \mathrm{kHz}$ is still containing weak components from the knocks of the loose parts and the background noise is surprisingly weak in that range $[2,4]$.

The test section where measurements have been made generally serves for cooling a shaker. The test section has two iron tubes: the cold leg and the hot leg (see Fig. 1). We installed 4 accelerometers on the cold leg. We generated internal and external events on the tube. For internal excitation we inserted different small objects in the tube. Due to turbulent flow, small parts fixed on a wire went on wild vibration knocking on inner wall of the tube. For external excitation we hit the outer side of the tube with an iron stack. 


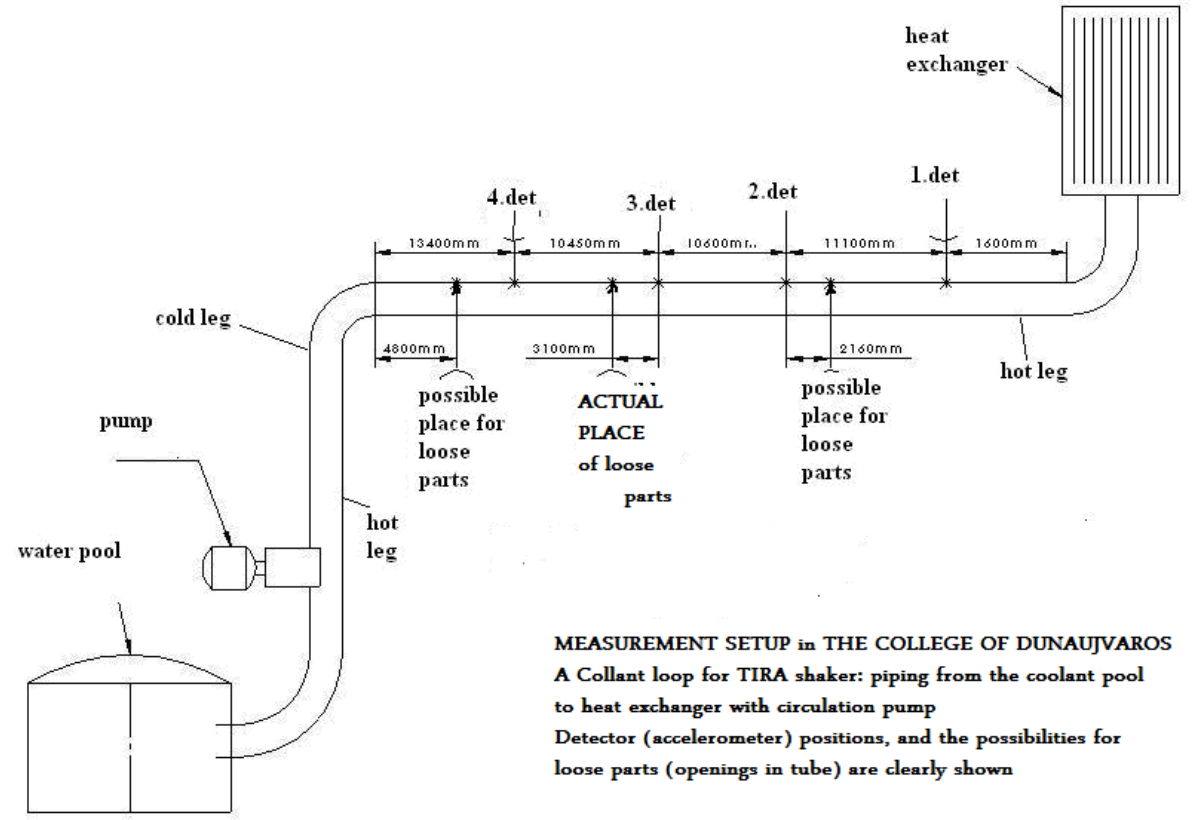

Figure 1: Measurement Setup

We put 3 different types of objects into the tube (Table 1 ):

- The first was the biggest one: a M10 iron bolt of $11 \mathrm{~g}$.

- The second was the middle sized one: M8 iron bolt of about $6 \mathrm{~g}$

- And the last one was a piece of Bakelite with weight of $1.7 \mathrm{~g}$.

There was only one object at one time inside the tube.

Table 1: Objects in the tube.

\begin{tabular}{l|c|c|c} 
Type & M10 bolt & M8 bolt & bakelite piece \\
\hline Mass & $11,16 \mathrm{~g}$ & $5,58 \mathrm{~g}$ & $1,67 \mathrm{~g}$ \\
\hline Photo & & &
\end{tabular}




\subsection{Measurement software}

Based on LABVIEW we developed a software for measurement, which can sample and store the signals of accelerometers up to 4 sensors in ASCII format specified by us.

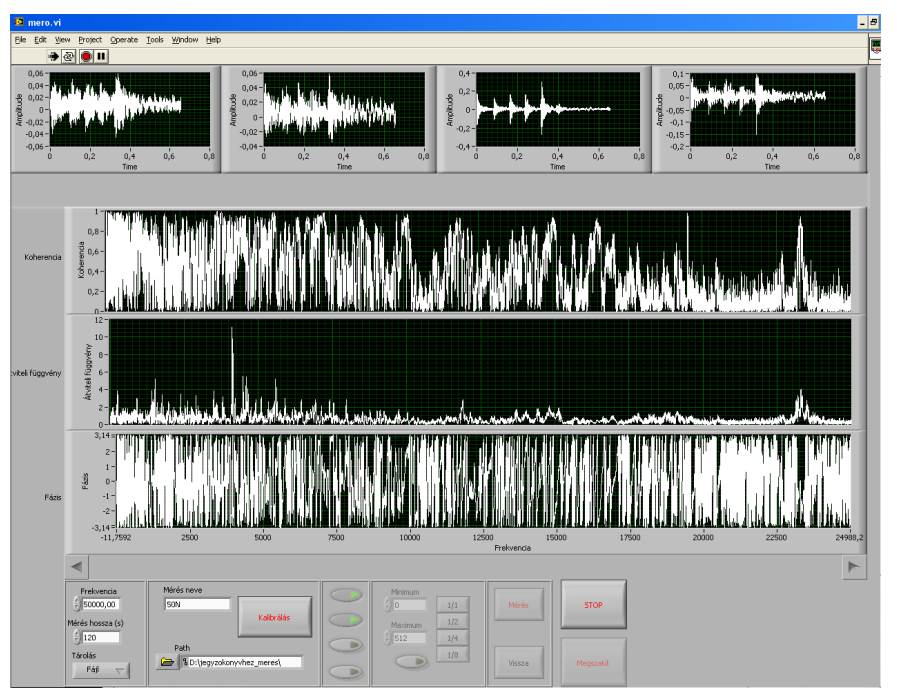

Figure 2: Display of measurement software during sampling

While measuring the program shows the time series, the coherence, the transfer function and the phase in real-time (cf. Fig. 2) for the operator. The user has to set the length of the measure, while a process bar shows the actual state of measuring. The software has three states:

Beginning state First the operator has to set the sampling frequency, the time length of measurement, the method of storage.

Calibrating state In the second step the user can select sensor pairs to see the cross functions. It is possible to zoom into special points of functions.

Measuring state If every sensor pairs has coherence peaks than the last step is the measuring. All the buttons are gray colored except the STOP button.

\section{Event Detection}

Once we did the record, we have to find and select events from the whole time series. Our preferred method is the Sequential Probability Ratio Test (SPRT), since it is generally accepted [5] and we also found that they are the most effective and reliable for this purposes. However, for carrying out the SPRT first we have to 
filter the signal to remove large background noise. We did that using Autoregressive (AR) filtering.

\subsection{Autoregressive filtering}

We build an autoregressive model using Durbins method on a record without acoustic events, to describe the background without the acoustic events.

The expression of autoregressive modeling is:

$$
y_{k}=\sum_{i=1}^{P} a_{i} y_{k-i}+w_{k}
$$

where $y$ are the sampled data, $a_{i}$ are the autoregressive coefficients, while the $w$ is a white, additional noise. It is supposed, that the autoregressive model will describe everything, what is deterministic in the background having in mind, that $P$ is the degree of freedom of the system. This AR model was used for filtering the actual records with events.

The formula of autoregression filtering:

$$
x_{k}^{\text {filtered }}=x_{k}^{\text {measured }}-\sum_{i=1}^{P} a_{i} x_{k-i}^{\text {measured }}
$$

This filtering method is very effective in real-time environment, because after the model has been built, i.e. the autoregressive coefficients have been estimated from the background measurements, the filtering can be made by subtracting the AR modeled data from the actually measured data.

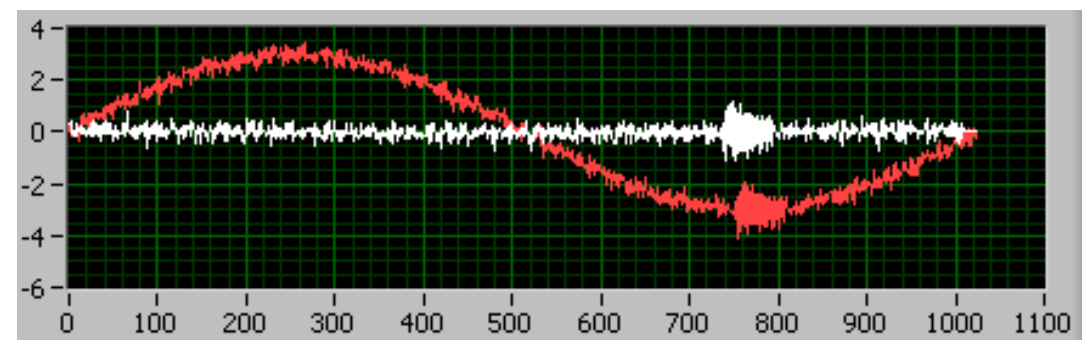

Figure 3: Test of autoregressive filtering

On Fig. 3 one may see a simulated example for autoregressive filtering. The red line shows a generated (simulated) signal: one period of sine function with added white Gaussian noise and one transient event (burst) on it. The white is the filtered signal. 


\subsection{Sequential Probability Ratio Test}

The next step is the Sequential Probability Ratio Test, which serves for event detection in the filtered signal. The theoretical formula of SPRT [2]:

$$
\lambda_{i}=\ln \left(\frac{p\left(x_{1}, x_{2}, \ldots, x_{i} \mid H_{1}\right)}{p\left(x_{1}, x_{2}, \ldots, x_{i} \mid H_{0}\right)}\right)
$$

The point-by-point formula of SPRT:

$$
\Delta \lambda_{i}=\lambda_{i}-\lambda_{i-1}=\ln \frac{f_{1}\left(x_{i} \mid H_{i}\right)}{f_{1}\left(x_{i} \mid H_{0}\right)}
$$

Lambda function (3) is a logarithm of a ratio of two conditioned probabilities. In the numerator we have the probability that the samples belong to the probability functions with condition $H_{1}$, i.e. the probability density function of that. While in the denominator we have the probability that the samples belong to the probability function conditioned by hypothesis $H_{0}$. If we substitute the normal distribution probability variable density function to the elementary lambda increment function of SPRT (4) then we get the following equation (5):

$$
\Delta \lambda_{i}=\frac{\sigma_{1}^{2}-\sigma_{0}^{2}}{2 \sigma_{1}^{2} \sigma_{0}^{2}} x_{i}^{2}-\ln \frac{\sigma_{1}}{\sigma_{0}}
$$

To take decision by the SPRT lambda function we need two parameters $A$ and $B$. When the measured signal similar in statistical sense to the background, i.e. when process can be described by Hypothesis $H_{0}$ the lambda function has a negative increment values and tends to go to negative infinity, step by step. We take decision, that the signal belongs to background type when the value of the lambda function exceeds value $A$ (becomes less than $A$ ). When the measured signal deviation is different from the background then lambda function has positive increment value and tends to raise. We take decision, when it exceeds value $B$.

$A$ can be calculated from the probability inequality [2]

$$
1-A F P \leq F A P \cdot e^{A}
$$

Expressing $A$ :

$$
A=\ln \frac{A F P}{1-F A P}
$$

$B$ can be calculated also from:

$$
1-A F P \geq F A P \cdot e^{B}
$$

Expressing $B$ :

$$
B=\ln \frac{1-A F P}{F A P}
$$

where $A F P$ means the Alarm Failure Probability and FAP means False Alarm Probability. 
Table 2: The $A$ and $B$ parameters

\begin{tabular}{r|r|r|r} 
AFP & FAP & $A$ & $B$ \\
\hline $10 \%$ & $10 \%$ & $-2,2$ & 2,2 \\
\hline $1 \%$ & $1 \%$ & $-4,6$ & 4,6 \\
\hline $0,1 \%$ & $0,1 \%$ & $-6,91$ & 6,91 \\
\hline $1 \%$ & $10 \%$ & $-4,5$ & 2,29 \\
\hline $0,1 \%$ & $10 \%$ & $-6,8$ & 2,3
\end{tabular}

Calculated [2] values for $A$ and $B$ using equation (7) and (9) are presented in Table 2 .

If the value of lambda hits one of the limits then SPRT takes a decision A or $\mathrm{B}$, where $\mathrm{A}$ means that, there was no any changes in the signal in comparison to the background, and B means that something has happened in the signal. After a decision has been taken we set the next lambda value to zero.

There is a trivial example on Fig. 4 showing how SPRT works. One can see the acoustic event in the upper time signal. SPRT shown in the bottom window goes down regularly exhibiting a saw tooth shape until there is only background noise in the time signal. At the beginning of the acoustic event lambda function will start to grow toward the positive values. However, this is a trivial case, here one does not need really the SPRT for event recognition.

There is a more realistic case on Fig. 5. The original sampled signal is shown in red color. The white signal in the upper window is that signal filtered by AR. Without the help of SPRT lambda function it is rather difficult to select real events, real burst even in the filtered signal. With SPRT events are clearly marked, and really one can see, that there is something in the filtered signal, which deviates at those time spots from the general behavior of the background. SPRT senses the standard deviation differences, where human eye doesnt see it at all.

We processed by this method all measured signals described in Section 1.

\section{Analyses of selected time sequences}

\subsection{Identification of the event using spectra}

The next task is to characterize the records classified by SPRT method into different classes. We calculated the averaged Power Spectrum Density function for backgrounds (A decisions of SPRT) and the events (B decisions of SPRT). They are shown on Figs. 6 and 7, respectively.

It is clearly seen on Fig. 6, that there are two peaks $a$ and $b$ sitting on the noise level when there was no event in the measurement. The noise level is falling with the growing frequency. We believe that peaks are due to eigenfrequencies of the tube, while the falling noise level is due to friction of the flow. They characterize 


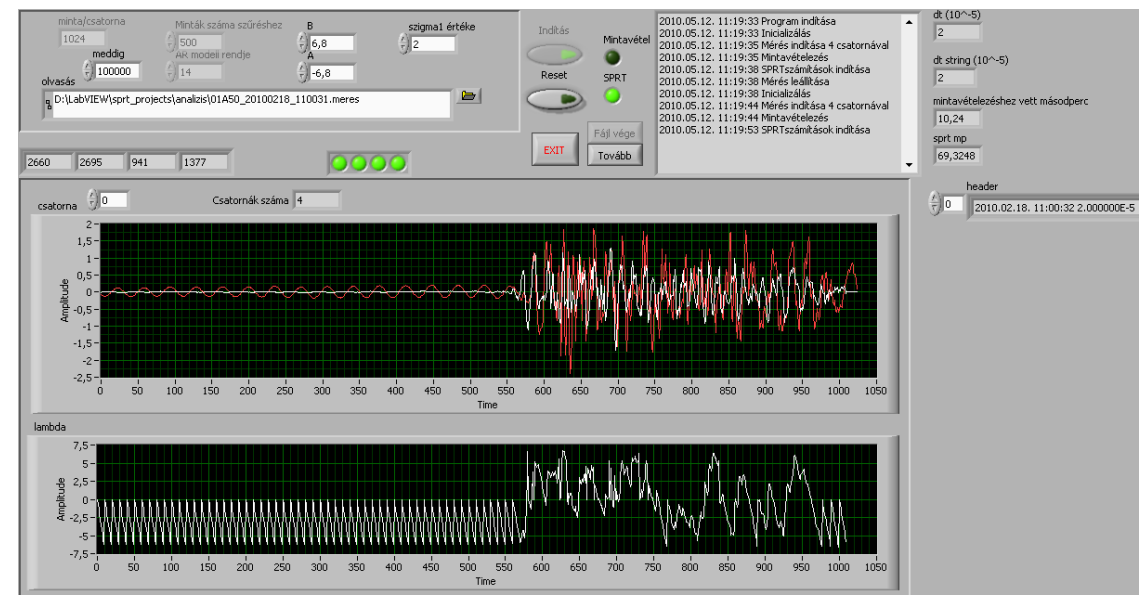

Figure 4: Trivial example

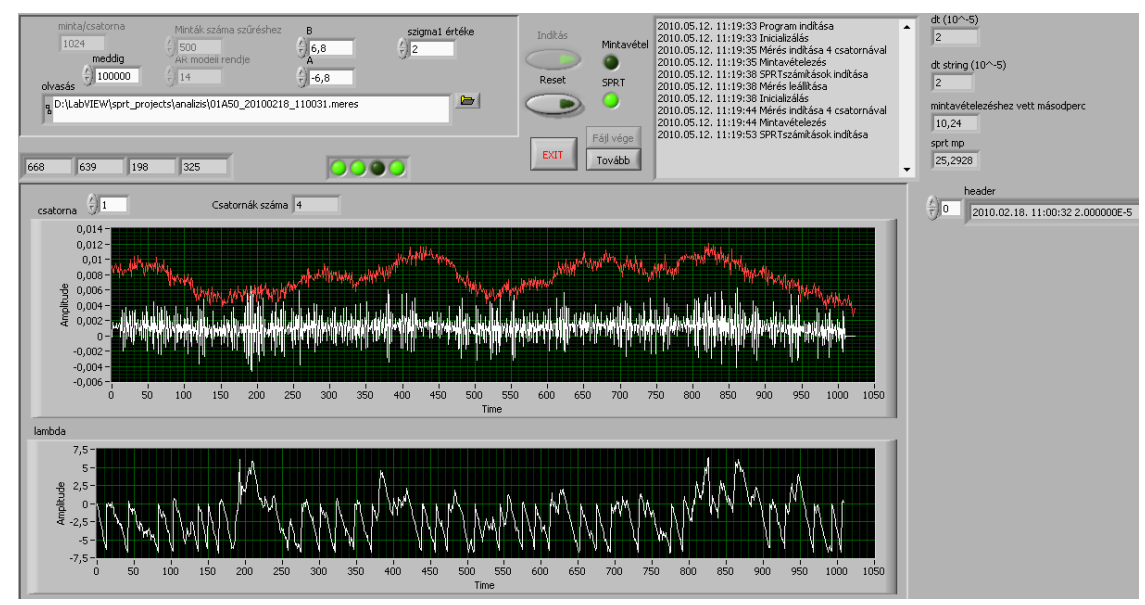

Figure 5: Real measurement example

the background noise of the measurements.

One can see $a$ and $b$ peaks and falling noise level from the background measurement in the average power spectrum density function of events as well (Fig. 7). But there are 3 other specific peaks (I,II,III) which are responsible for the events.

The numerical difference between the spectrum of events and the spectrum of noises (Fig. 8), shows the specific descriptors of the event. The smaller peaks are due to uncertainties in standard deviation of the measured signal, but the bigger peaks marked on figure are useful for identification purposes. 


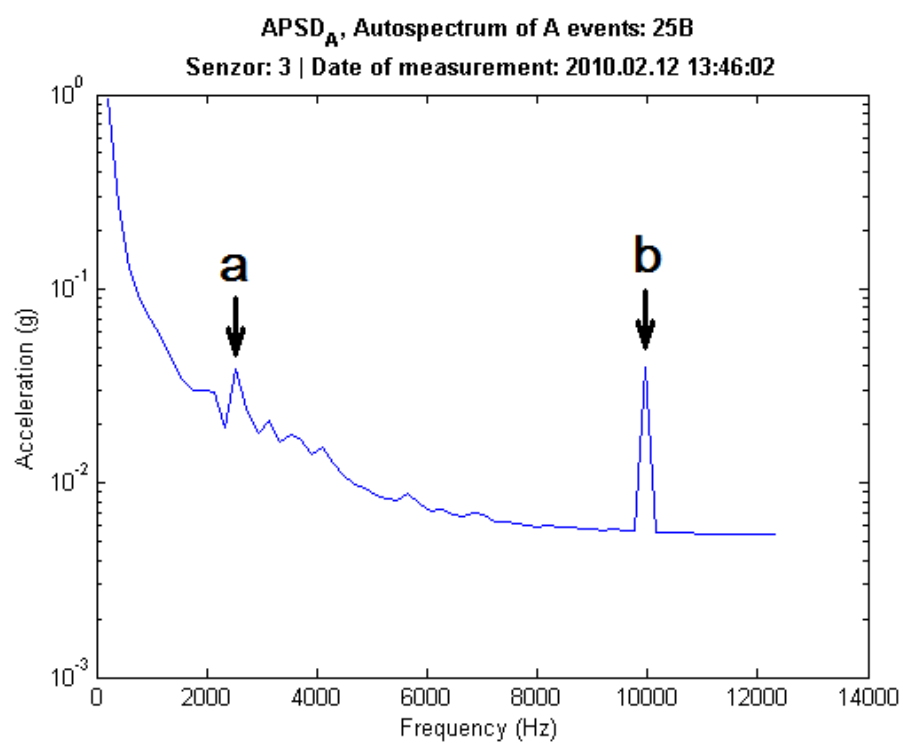

Figure 6: The averaged Power Spectrum Density function of background

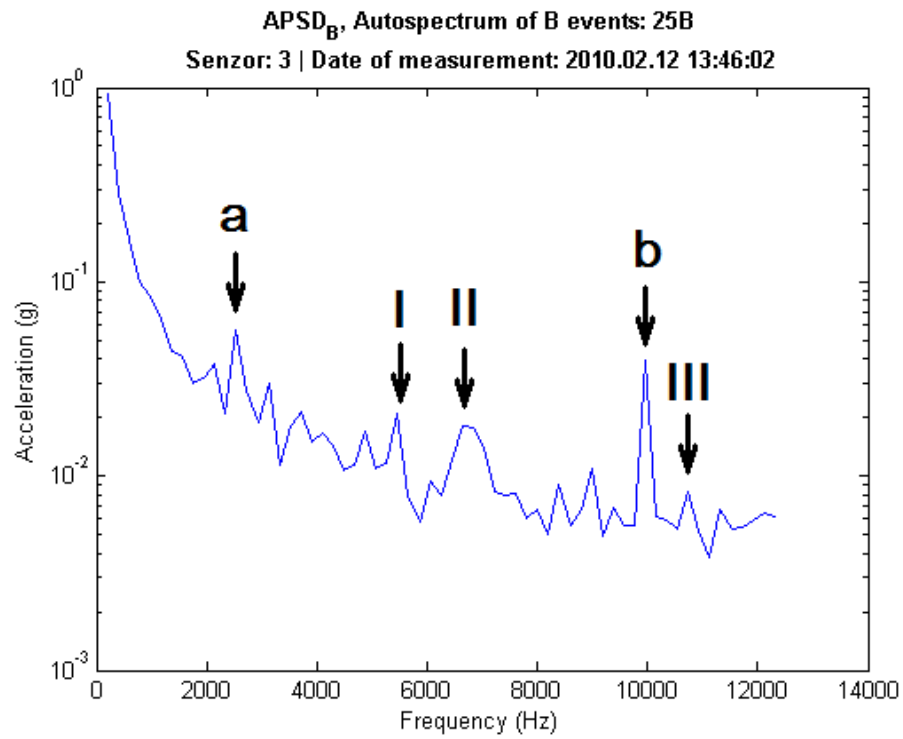

Figure 7: Averaged Power Spectrum Density function of acoustic events, what selected by SPRT. 


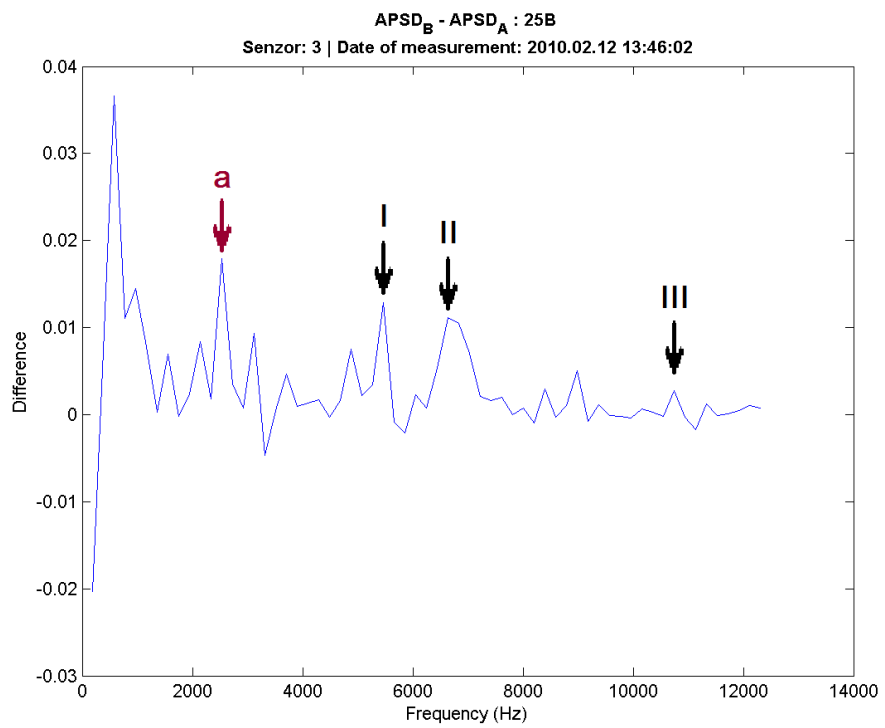

Figure 8: The numerical difference between the two spectrums, B-A.

\subsection{Localization}

The simplest method for localization is based on the front of the events identified by SPRT. There are two cases clearly depicted on Figs. 9 and 10. The impact can be either outside or inside of the section determined by the two sensors.

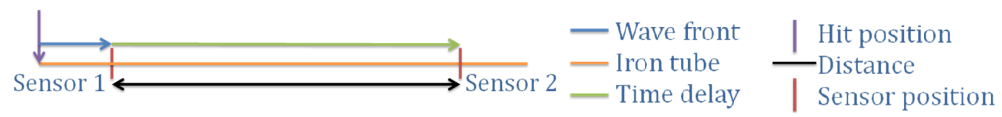

Figure 9: Hit outside the analyzed interval.

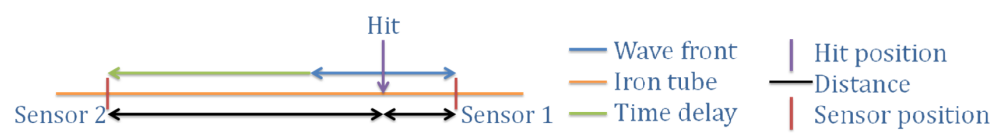

Figure 10: Hit intside the analyzed interval.

On these figures we used the following notations:

- The orange line is the tube itself.

- The purple arrow shows the point of hits. 
- The blue arrows show the propagation of the front of wave until it arrives to the first sensor.

- The green arrows show the propagation after the front has passed the first sensor. It is the time difference. It is the time delay.

- The black line shows the known distances in Fig. 9 and the unknown distances in Fig. 10.

For localization procedure we have to estimate the time delays between the events measured by different sensors.

\subsubsection{Method using Cross Correlation Function (CCF)}

In case of traditional localization one can calculate the Cross Correlation Function (Fig. 11) to estimate the time delay and thus to localize the hits. The argument of maximum of this function shows the time delay between the two signals.

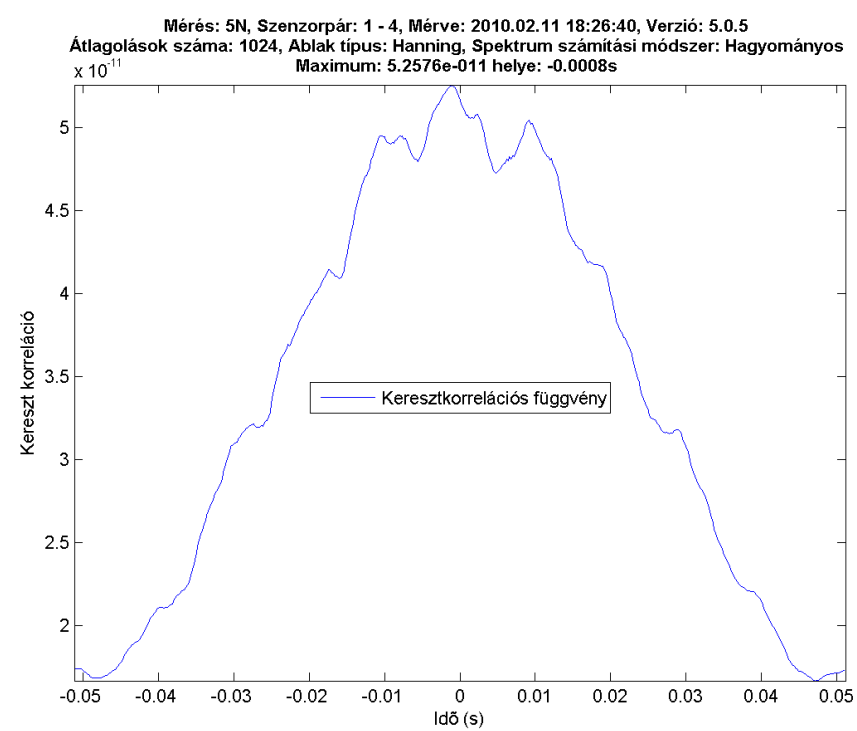

Figure 11: Example CCF

\subsubsection{Method using Impulse Response Function (IRF)}

There is another function in traditional localization procedures [3]: one can calculate the Impulse Response Function (Fig. 11) to estimate time delays and thus to localize the hits. The argument of maximum of this function shows the time delay between the two signals, like the cross correlation function. This method is 
better in the sense that it may show also the velocity distribution, but it has higher uncertainties.

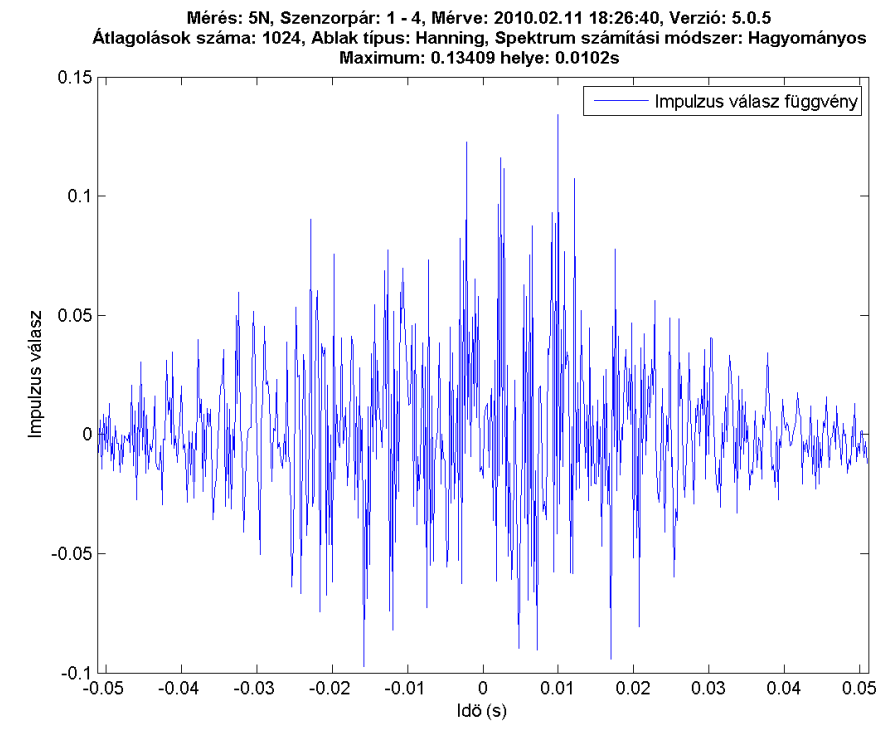

Figure 12: Example of IRF

\subsubsection{New method}

The new way of localization is to use learning algorithms. We hit the tube several times on the marked points on Fig. 13. Then we calculate different description parameters from power spectrums to train the learning algorithm (for example neural network).

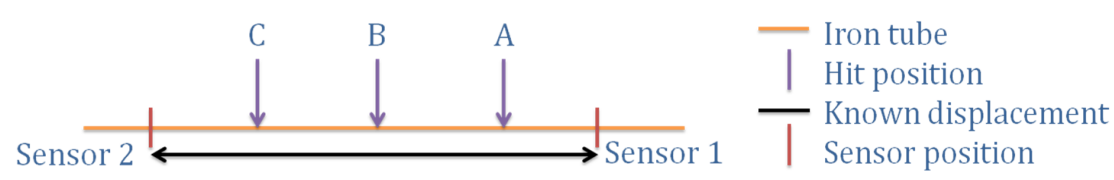

Figure 13: Localization with identification 


\section{Identification}

\subsection{Traditional way}

To distinguish the material of the hitting object at the beginning we applied the spectrum division method proposed in [7]. We divide the power spectrum of acoustic events into four parts (see Fig. 14).

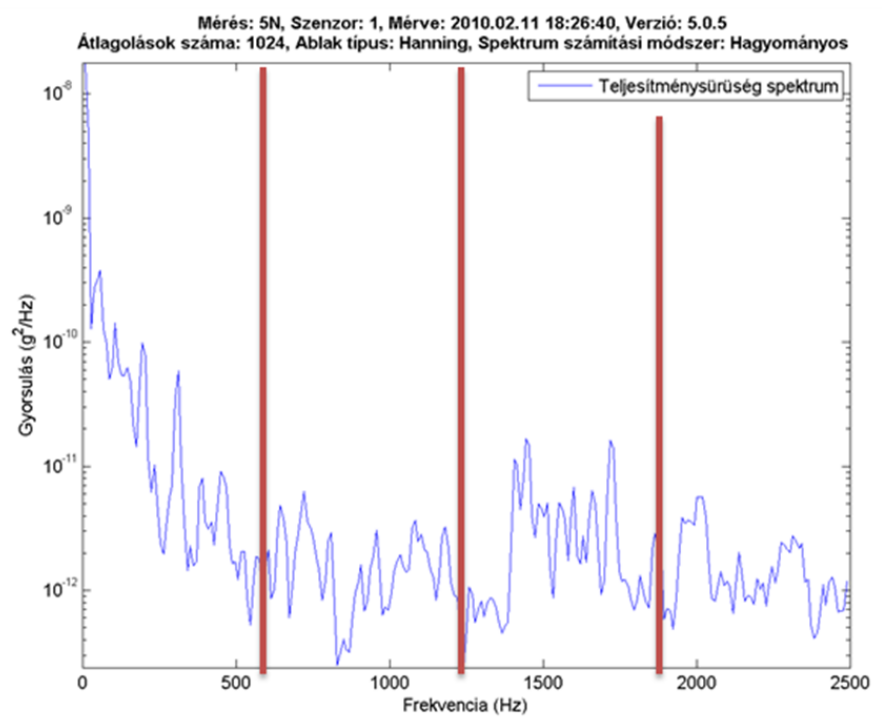

Figure 14: Spectrum dividing method

Using this spectrum division we can estimate their RMS Root Mean Square value by formula:

$$
R M S_{i}=\Delta f \times \sum_{j=(i-1)(n / 4)}^{i \cdot(n / 4)-1} A P S D_{j}
$$

In this way, we have got 4 numbers, which characterize the actual event. This method is insensitive for the position of peaks. However, the peaks contain important information. For example cutting a peak into two parts can produce big mistake in this method.

\subsection{New method}

We are looking for methods to describe spectra in a more effective way using machine learning processes.

We are trying to calculate the skeleton of spectrums (Fig. 15). The endpoints of skeletons are positive peaks that we are interested in. We borrowed the skeleton 


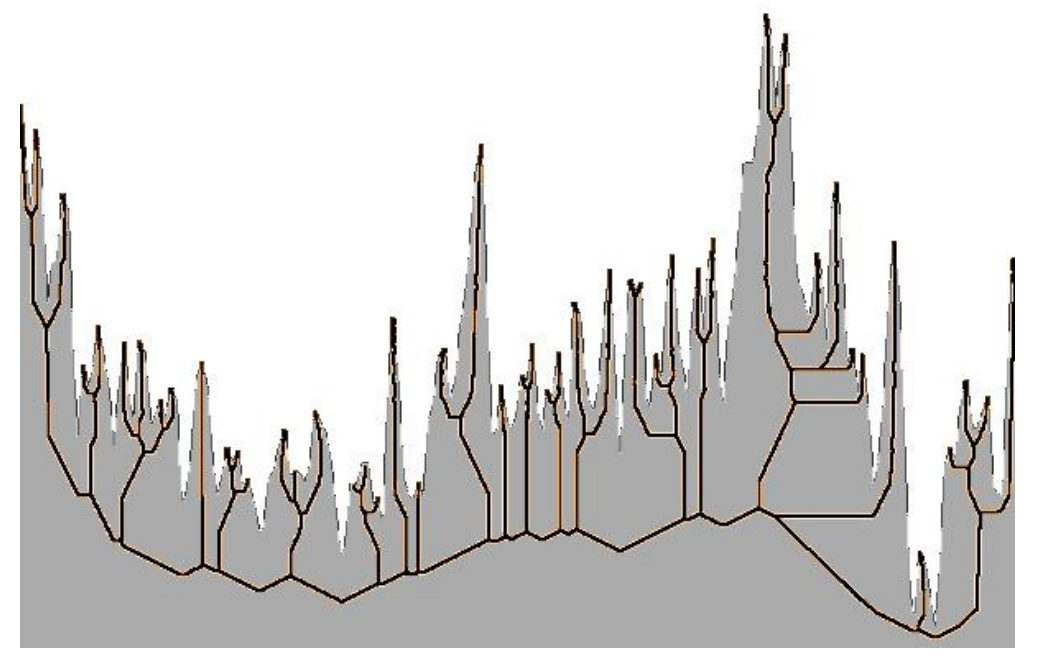

Figure 15: Skeleton of a spectrum

method and program from Gábor Németh [8]. It produces skeleton which has different nodes and different distribution of the branches. In the future we shall use these parameters for characterizing the event.

\section{$5 \quad$ Summary and Conclusions}

The fact that sequential probability ratio test is one of the best methods for identifying abnormal events had been proposed and demonstrated earlier cf. [1, 2, 3, 4, 5]. However, earlier only offline data evaluation programs have been used to select events in high background noise. The presented LABVIEW program opens the way for on-line application of the SPRT method for event selection. A benchmark measurement has been evaluated to show the capability of the event selection method. Efficiency of the method was clearly demonstrated in our paper. Spectral estimation of selected methods clearly pointed at differences of spectral components, when different object impacted the wall. This opens the route for object identification using autospectrum. However, it is not easy to automotive the spectrum patter recognition having several different peaks in the spectrum. We are involving the skeleton method. For the other important task, for the localization of the impact place we tried three methods: we used time delay of the fronts of the events identified by SPRT; we used the cross correlation and the impulse response estimation to retrieve the time delay. The front cannot be estimated very precisely; the cross-correlation seems to be too wide for precise time delay estimation; the impulse response has rather large uncertainty. We tried a new method based on neural network, which seems to be very promising. 


\section{References}

[1] Szappanos, G., Feher, A., Lorincz, J.,Nemes, L., Por, G., Csikos, T., Glodi, O., Lipcsei S., Tri, T. D. A New Digital Expert Loose Part Detection System. Annals in Nuclear Energy, 24(14):1097-1103, 1997

[2] Szappanos, G., Por,G. Improvements in the Theory of Identification of BurstShaped Events for Fault Diagnosis. Nuclear Science and Engineering, 13:261267, 1999

[3] Por, G., Szappanos, G. Advanced Loose Part Monitoring System for Nuclear Power Plants. Proc. of International Conference Nuclear Energy in Central Europe 2000

[4] Por, G. ,Szappanos, G. ALPS, Advanced Loose Parts System for Paks NPP. Proc. of International Conference Nuclear Energy in Central Europe 2001

[5] Schoonewelle, H., Van Der Hagen, T. H. J. J., and Hoogenboom, J. E. A comparison of three time-domain anomaly detection methods. Ann. Nucl. Energy, 23(2):159-170, 1996

[6] Por, G., Berta, M., Csuvar, M. Measurement of the coolant flow rate using correlation of temperature fluctuations. Progress in Nuclear Energy, Proccedings of the 8th Symposium on Nuclear Reactor Surveillance and Diagnostics, 43(1-4):281-288, 2003

[7] Tsunodaa, T., Kato, T., Hirata, K., Sekido, Y., Sendai, K., Segawa, M., Yamatoku, S., Morioka, T., Sanoa, K., and Tsuneokaa, O. Studies on the loose part evaluation technique. Progress in Nuclear Energy 15:569-576, 1985

[8] Kardos, P., Németh, G., and Palágyi, K. An OrderIndependent Sequential Thinning Algorithm. Wiederhold, P. and Barneva, R. P., editors, IWCIA 2009, LNCS 5852 pages 162-175, 2009 